\title{
Further study of the global minimum constraint on the two-Higgs-doublet models: LHC searches for heavy Higgs bosons
}

\author{
Ning Chen, ${ }^{1, \dagger}$ Chun Du, ${ }^{2, *}$ Yongcheng Wu, ${ }^{3, \$}$ and Xun-Jie $\mathrm{Xu}^{4, \S}$ \\ ${ }^{1}$ School of Physics, Nankai University, Tianjin 300071, China \\ ${ }^{2}$ Key Laboratory of Cosmic Rays (Tibet University), Ministry of Education, Lhasa 850000, Tibet, China \\ ${ }^{3}$ Ottawa-Carleton Institute for Physics, Carleton University, 1125 Colonel By Drive, \\ Ottawa, Ontario K1S 5B6, Canada \\ ${ }^{4}$ Max-Planck-Institut für Kernphysik, Postfach 103980, D-69029 Heidelberg, Germany
}

(Received 18 October 2018; revised manuscript received 17 January 2019; published 11 February 2019)

\begin{abstract}
The usually considered vacuum of the two-Higgs-doublet model (2HDM) could be unstable if it locates at a local but not global minimum (GM) of the scalar potential. By requiring the vacuum to be a GM, we obtain an additional constraint, namely the GM constraint, on the scalar potential. In this work, we explore the GM constraint on the $C P$-conserving general $2 \mathrm{HDM}$. This constraint is found to put limits on the soft $\mathbb{Z}_{2}$ breaking mass parameter $m_{12}^{2}$ and also squeeze the heavy $C P$-even Higgs boson mass into larger values for the $m_{12}^{2}<0$ case. Combined with the current global signal fits from the LHC measurements of the $125 \mathrm{GeV}$ Higgs boson, we discuss the phenomenological implications for the heavy Higgs boson searches at the LHC.
\end{abstract}

DOI: $10.1103 /$ PhysRevD.99.035011

\section{INTRODUCTION}

In the studies of new physics beyond the Standard Model (BSM), it is quite often that one has an extended Higgs sector. A simple and well-known example is the twoHiggs-doublet model (2HDM), ${ }^{1}$ which was motivated from several different aspects, such as supersymmetry [2,3], $C P$ violation [4], and axion models [5]. With an additional Higgs doublet introduced, the Higgs potential in the 2HDM may develop several different minima. Therefore, one may encounter the possibilities as follows: (i) one Higgs doublet does not acquire a vacuum expectation value (VEV), (ii) the Higgs VEVs break the $C P$ symmetry, or (iii) the Higgs VEVs even break the U(1) $)_{\mathrm{EM}}$ symmetry. It has previously been studied in Refs. [6-25] that several minima can coexist in the $2 \mathrm{HDM}$ potential so that the desired vacuum might be a local minimum that could decay into a deeper one through quantum tunneling [26,27], causing instability of the desired vacuum.

\footnotetext{
*Corresponding author. duchun@utibet.edu.cn

†chenning_symmetry@nankai.edu.cn

*ycwu@ physics.carleton.ca

\unjie@mpi-hd.mpg.de

${ }^{1}$ See Ref. [1] for a comprehensive review.

Published by the American Physical Society under the terms of the Creative Commons Attribution 4.0 International license. Further distribution of this work must maintain attribution to the author(s) and the published article's title, journal citation, and DOI. Funded by SCOAP.
}

To avoid the vacuum instability, one may impose a global minimum (GM) condition for the desired vacuum. This leads to new constraints on the Higgs potential, in addition to the conventional bounded-from-below (BFB) constraints and the unitarity bounds. Recently, the GM condition of the $2 \mathrm{HDM}$ potential has been analytically formulated in Ref. [28] and tentatively applied to constrain the general 2HDM. It has been demonstrated that the GM constraint can sometimes be robust in constraining the parameter space of the $2 \mathrm{HDM}^{2}$

In this work, we further study the GM constraint on the 2HDM, with the focus on the phenomenological implications at the LHC. It turns out that the GM condition is likely to put constraints on the masses of heavy Higgs bosons and the soft $\mathbb{Z}_{2}$ breaking scale of $m_{12}^{2}$ in the 2HDM. In turn, these constraints are directly connected to the Higgs self-couplings in the 2HDM. From the experimental point of view, the Higgs self-couplings are likely to be probed by the high-luminosity (HL) and/or highenergy (HE) LHC runs, by looking for the Higgs boson pair productions. Since the discovery of the $125 \mathrm{GeV}$ Higgs boson at the LHC, a lot of efforts have been made in probing such processes in different new physics models at

\footnotetext{
${ }^{2}$ Typically in many BSM models with scalar extensions, the GM conditions of the desired vacua can be nontrivial and deserve further studies. The GM conditions of some models have been studied before, such as the Georgi-Machacek model in Ref. [29], the Type II Seesaw model in Ref. [30], and the left-right symmetric model in Ref. [31].
} 
the LHC [32-49]. Since the Higgs self-couplings in the $2 \mathrm{HDM}$ can determine the corresponding partial decay widths of a heavy Higgs boson into lighter Higgs pairs, the future experimental searches for heavy Higgs bosons in the 2HDM may also be sensitive to the GM constraint.

The layout of this paper is described as follows. In Sec. II, we revisit the $C P$-conserving general $2 \mathrm{HDM}$, where we put emphasis on the GM constraint on the 2HDM potential. This constraint, together with the usual tree-level BFB and perturbative unitarity constraints, will be imposed on the 2HDM parameter space. In Sec. III, we consider benchmark models in two different scenarios, namely, the degenerate heavy Higgs boson scenario of $M_{A}=M_{H}=M_{ \pm}$and the heavy Higgs boson spectrum involving exotic decays. It turns out that the GM condition leads to additional restrictions on the parameter space. In Sec. IV, we study the LHC phenomenologies based on the GM constraints on the benchmark models. Since the GM constraint on the $m_{12}^{2}$ parameter will control the Higgs boson self-couplings in the 2HDM, the pair productions of both SM-like and BSM Higgs bosons at the LHC can be relevant to this constraint. The current LHC $13 \mathrm{TeV}$ searches for the Higgs boson pairs, as well as other exotic heavy Higgs boson decay modes, are imposed to the benchmark models with the GM constraint taken into account. Finally, we conclude in Sec. V.

\section{THE GENERAL 2HDM AND THE GM CONSTRAINT}

\section{A. The general 2HDM}

The scalar potential of the general $2 \mathrm{HDM}$ is written as follows:

$$
\begin{aligned}
V\left(\Phi_{1}, \Phi_{2}\right)= & m_{11}^{2}\left|\Phi_{1}\right|^{2}+m_{22}^{2}\left|\Phi_{2}\right|^{2}-m_{12}^{2}\left(\Phi_{1}^{\dagger} \Phi_{2}+\text { H.c. }\right) \\
& +\frac{1}{2} \lambda_{1}\left(\Phi_{1}^{\dagger} \Phi_{1}\right)^{2}+\frac{1}{2} \lambda_{2}\left(\Phi_{2}^{\dagger} \Phi_{2}\right)^{2}+\lambda_{3}\left|\Phi_{1}\right|^{2}\left|\Phi_{2}\right|^{2} \\
& +\lambda_{4}\left|\Phi_{1}^{\dagger} \Phi_{2}\right|^{2}+\frac{1}{2} \lambda_{5}\left[\left(\Phi_{1}^{\dagger} \Phi_{2}\right)^{2}+\text { H.c. }\right],
\end{aligned}
$$

where all the couplings are real for the $C P$-conserving case. Here, we do not include the $\mathbb{Z}_{2}$ broken terms of $\lambda_{6}\left(\Phi_{1}^{\dagger} \Phi_{1}\right)$ $\left(\Phi_{1}^{\dagger} \Phi_{2}\right)+\lambda_{7}\left(\Phi_{2}^{\dagger} \Phi_{2}\right)\left(\Phi_{1}^{\dagger} \Phi_{2}\right)+$ H.c., focusing our study on the potential with softly broken $\mathbb{Z}_{2}$ symmetry.

The potential in Eq. (1) contains eight parameters, namely $m_{11}^{2}, m_{12}^{2}, m_{22}^{2}$, and $\lambda_{1 \ldots 5}$, which are usually referred to as the parameters in the generic basis. In phenomenological studies, it is usually more convenient to work in the so-called physical basis, including the five physical boson masses of $\left(M_{h}, M_{H}\right.$, $\left.M_{A}, M_{ \pm}\right)$, two mixing angles of $(\alpha, \beta)$, a soft $\mathbb{Z}_{2}$ broken mass squared term of $m_{12}^{2}$, and the electroweak VEV $v \approx 246 \mathrm{GeV}$.

In the general 2HDM, there could be tree-level flavorchanging neutral currents (FCNC), which are well-known constraints on such model. To alleviate the tree-level FCNC process constraints, the SM fermions of a given representation are usually assigned to a single Higgs doublet.
We focus on the so-called Type I and Type II Yukawa couplings of

$$
\mathcal{L} \supset \sum_{h_{i}=h, H}-\frac{m_{f}}{v}\left(\xi_{i}^{f} \bar{f} f h_{i}+\xi_{A}^{f} \bar{f} i \gamma_{5} f A\right),
$$

with

$$
\text { Type I: } \begin{aligned}
\xi_{h}^{f} & =\sin (\beta-\alpha)+\frac{\cos (\beta-\alpha)}{\tan \beta}, \\
\xi_{H}^{f} & =\cos (\beta-\alpha)-\frac{\sin (\beta-\alpha)}{\tan \beta}, \\
\xi_{A}^{u} & =\frac{1}{\tan \beta}, \quad \xi_{A}^{d, \ell}=-\frac{1}{\tan \beta},
\end{aligned}
$$

$$
\text { Type II: } \begin{aligned}
\xi_{h}^{u} & =\sin (\beta-\alpha)+\frac{\cos (\beta-\alpha)}{\tan \beta}, \\
\xi_{h}^{d, \ell} & =\sin (\beta-\alpha)-\cos (\beta-\alpha) \tan \beta, \\
\xi_{H}^{u} & =\cos (\beta-\alpha)-\frac{\sin (\beta-\alpha)}{\tan \beta}, \\
\xi_{H}^{d, \ell} & =\cos (\beta-\alpha)+\sin (\beta-\alpha) \tan \beta, \\
\xi_{A}^{u} & =\frac{1}{\tan \beta}, \quad \xi_{A}^{d, \ell}=\tan \beta .
\end{aligned}
$$

Besides, two $C P$-even Higgs bosons couple to the gauge bosons such that

$$
\mathcal{L} \supset \sum_{h_{i}=h, H} a_{i}\left(2 \frac{m_{W}^{2}}{v} W_{\mu}^{+} W^{-\mu}+\frac{m_{Z}^{2}}{v} Z_{\mu} Z^{\mu}\right) h_{i},
$$

with

$$
a_{h}=\sin (\beta-\alpha), \quad a_{H}=\cos (\beta-\alpha) .
$$

The current LHC run I and run II have measured the signal strengths of the SM-like $125 \mathrm{GeV}$ Higgs boson ${ }^{3}$ via different channels [50-78]. Here, they are combined to obtain the $95 \%$ C.L. regions in the $\cos (\beta-\alpha)$ vs $\tan \beta$ plane, as shown in Fig. 1. In both Type I and Type II, the alignment limit of $\beta-\alpha=\pi / 2$ is favored by the global fit. For the Type I $2 \mathrm{HDM}$ with $\tan \beta \gtrsim 2,|\cos (\beta-\alpha)|$ is constrained to be less than about 0.4 with the LHC run-I and run-II data. This is envisioned to be further constrained to be less than 0.2 with the HL-LHC runs in Ref. [79]. For the Type II 2HDM, large/small $\tan \beta$ inputs will enhance the Yukawa couplings $\xi_{h}^{d, \ell} / \xi_{h}^{u}$. Thus, the region around $\tan \beta=1$ accommodates the largest deviation from the alignment. The current LHC run-I and run-II measurements constrain $\cos (\beta-\alpha)$ in the range of $(-0.01,0.08)$

\footnotetext{
${ }^{3}$ Throughout the context, we always assume that $M_{h}=$ $125 \mathrm{GeV}$, while all other Higgs bosons are heavier.
} 
Type I

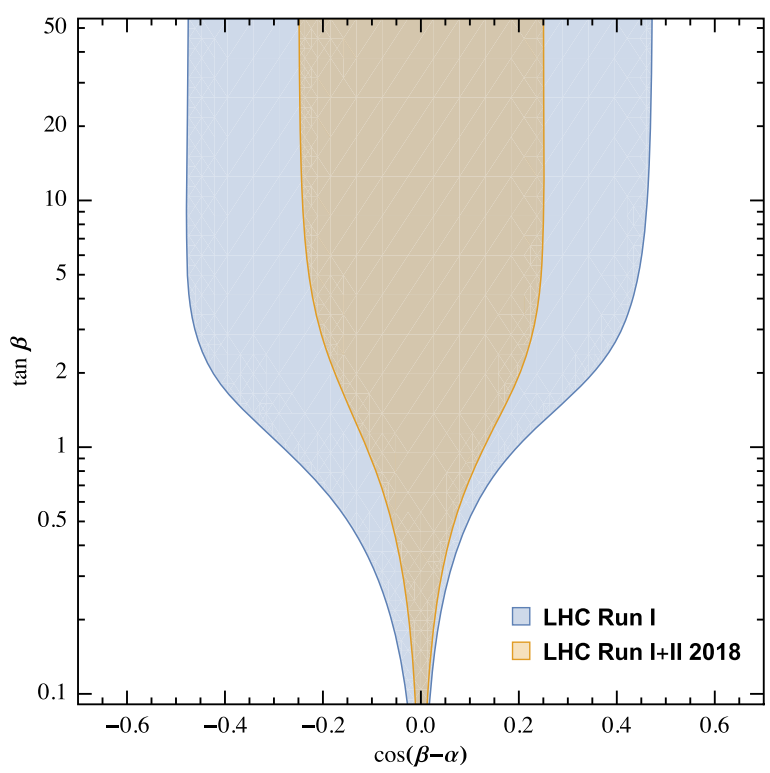

Type II

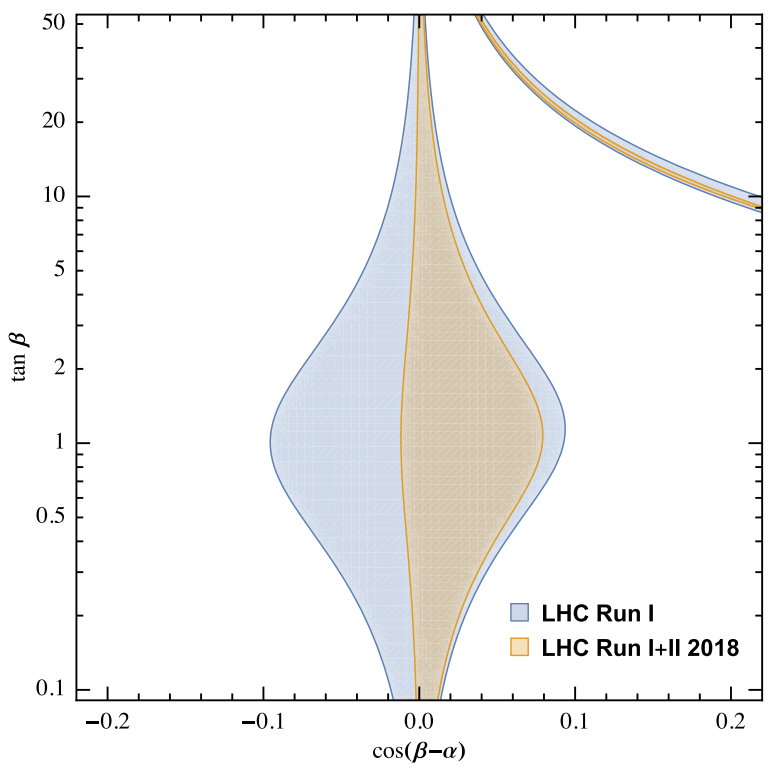

FIG. 1. The combined LHC run-I and run-II constraints on the $125 \mathrm{GeV}$ Higgs boson signal strengths in terms of $2 \mathrm{HDM}$ parameters $\cos (\beta-\alpha)$ and $\tan \beta$. Both Type I (left) and Type II (right) cases are displayed.

approximately with $\tan \beta=1$ (except for the wrong-sign Yukawa coupling region $[80,81])$.

\section{B. The GM constraint on the $2 \mathrm{HDM}$ potential}

As explained in the Introduction, to guarantee the absolute stability of the usually considered vacuum, we shall impose the GM constraint on the potential. First, we will present all the possible minima of the potential at the tree level and discuss the condition of the desired one being a global minimum. We realize that loop corrections can also have important influence on the GM constraint. Subsequently, we will also address the issue of including loop corrections.

At the tree level, by defining the following three $\mathrm{SU}(2)_{L}$ invariants of

$$
\begin{aligned}
q_{1} & \equiv\left|\Phi_{1}\right|^{2}=\Phi_{1}^{\dagger} \Phi_{1}, \quad q_{2} \equiv\left|\Phi_{2}\right|^{2}=\Phi_{2}^{\dagger} \Phi_{2}, \\
z & \equiv \Phi_{1}^{\dagger} \Phi_{2}=|z| e^{i \theta},
\end{aligned}
$$

the potential can be rewritten as

$$
\begin{aligned}
V\left(\Phi_{1}, \Phi_{2}\right)= & m_{11}^{2} q_{1}+m_{22}^{2} q_{2}-\left(m_{12}^{2} z+\text { H.c. }\right)+\frac{1}{2} \lambda_{1}\left(q_{1}\right)^{2} \\
& +\frac{1}{2} \lambda_{2}\left(q_{2}\right)^{2}+\lambda_{3} q_{1} q_{2}+\lambda_{4}|z|^{2} \\
& +\frac{1}{2}\left(\lambda_{5} z^{2}+\text { H.c. }\right) .
\end{aligned}
$$

In principle, one can directly minimize the above potential with respect to $q_{1}, q_{2}$, and $z$. However, one should notice that by definition, the three $\mathrm{SU}(2)_{L}$ invariants of $\left(q_{1}, q_{2}, z\right)$ satisfy the boundary conditions of

$$
q_{1,2} \geq 0, \quad q_{1} q_{2} \geq|z|^{2}
$$

Depending on whether the minima are on one of the boundaries in Eq. (8), we can classify the minima into five types, namely,

Type A: $q_{1}>0, \quad q_{2}>0, \quad q_{1} q_{2}>|z|^{2}$,

Type B: $q_{1}=0, \quad q_{2}>0, \quad z=0$,

Type C: $q_{1}>0, \quad q_{2}=0, \quad z=0$,

Type D: $q_{1,2}>0, \quad q_{1} q_{2}=|z|^{2}$,

Type E: $q_{1,2}=z=0$,

where, e.g., Type A is not on any of the boundaries and Type $\mathrm{E}$ is on all of the boundaries. All of the five types of minima have been solved in Ref. [28] and summarized in Table I.

The row in Table I containing the explicit forms of $\left\langle\phi_{1}\right\rangle$ and $\left\langle\phi_{2}\right\rangle$ indicates that Type D is the usually desired vacuum of $2 \mathrm{HDM}$. Type A minima could break $\mathrm{U}(1)_{\mathrm{EM}}$, while Type $\mathrm{B}$ and Type $\mathrm{C}$ minima appear in the so-called inert 2HDM. Type $\mathrm{E}$ is a trivial solution that is listed here for completeness. 
TABLE I. All possible local minima of the scalar potential. " $x$ " denotes a nonzero component, and " $*$ " stands for an arbitrary value (can be zero or nonzero).

\begin{tabular}{|c|c|c|c|c|c|}
\hline & Type A & Type B & Type C & Type D & Type E \\
\hline$\left\langle\phi_{1}\right\rangle,\left\langle\phi_{2}\right\rangle$ & {$\left[\begin{array}{l}0 \\
\times\end{array}\right],\left[\begin{array}{l}\times \\
*\end{array}\right]$} & {$\left[\begin{array}{l}0 \\
0\end{array}\right],\left[\begin{array}{l}0 \\
\times\end{array}\right]$} & {$\left[\begin{array}{l}0 \\
\times\end{array}\right],\left[\begin{array}{l}0 \\
0\end{array}\right]$} & {$\left[\begin{array}{l}0 \\
x\end{array}\right],\left[\begin{array}{l}0 \\
x\end{array}\right]$} & {$\left[\begin{array}{l}0 \\
0\end{array}\right],\left[\begin{array}{l}0 \\
0\end{array}\right]$} \\
\hline$\left(q_{1}, q_{2}, z\right)$ & Eq. (10) & $\left(0,-\frac{m_{22}^{2}}{\lambda_{0}}, 0\right)$ & $\left(-\frac{m_{11}^{2}}{\lambda_{1}}, 0,0\right)$ & Eq. (13) & $(0,0,0)$ \\
\hline Existence Condition & $q_{1}, q_{2}>0|z|^{2}<q_{1} q_{2}$ & $q_{2}>0$ & $q_{1}>0$ & $q_{1}, q_{2}>0|z|^{2}=q_{1} q_{2}$ & $/^{\mathrm{a}}$ \\
\hline$V_{\min }$ & Eq. (12) & $-\frac{m_{22}^{4}}{2 \lambda_{2}}$, Eq. (15a) & $-\frac{m_{11}^{4}}{2 \lambda_{1}}$, Eq. (15b) & Eq. $(15 c)$ & 0 \\
\hline
\end{tabular}

${ }^{\mathrm{a}}$ Not required.

The solution of Type A is given by

$$
\text { Type A: }\left(\begin{array}{c}
q_{1} \\
q_{2} \\
z \\
z^{*}
\end{array}\right)=\Lambda^{-1} b
$$

where

$$
\Lambda=\left(\begin{array}{cccc}
\lambda_{1} & \lambda_{3} & 0 & 0 \\
\lambda_{3} & \lambda_{2} & 0 & 0 \\
0 & 0 & \lambda_{5} & \lambda_{4} \\
0 & 0 & \lambda_{4} & \lambda_{5}^{*}
\end{array}\right), \quad b=\left(\begin{array}{c}
-m_{11}^{2} \\
-m_{22}^{2} \\
m_{12}^{2} \\
\left(m_{12}^{2}\right)^{*}
\end{array}\right)
$$

And the corresponding potential minimum is

$$
\begin{aligned}
V_{\min , \mathrm{A}}= & -\frac{1}{2} b^{T} \Lambda^{-1} b=\frac{-m_{11}^{4} \lambda_{2}-m_{22}^{4} \lambda_{1}+2 m_{11}^{2} m_{22}^{2} \lambda_{3}}{2\left(\lambda_{1} \lambda_{2}-\lambda_{3}^{2}\right)} \\
& -\frac{\left(m_{12}^{2}\right)^{2}}{\lambda_{4}+\lambda_{5}} .
\end{aligned}
$$

The Type $\mathrm{D}$ minimum is determined by $\partial V / \partial q_{1,2}=0$ : $m_{11}^{2}=m_{12}^{2} \tan \beta-\left[\lambda_{1} \cos ^{2} \beta+\left(\lambda_{3}+\lambda_{4}+\lambda_{5}\right) \sin ^{2} \beta\right] q$,

$$
m_{22}^{2}=m_{12}^{2} / \tan \beta-\left[\lambda_{2} \sin ^{2} \beta+\left(\lambda_{3}+\lambda_{4}+\lambda_{5}\right) \cos ^{2} \beta\right] q,
$$

where $q \equiv q_{1}+q_{2}$. Given the potential parameters of $\left(m_{11}^{2}, \lambda_{1}, \lambda_{2}, \ldots\right)$, Eqs. (13a) and (13b) can be solved with respect to $q$ and $\beta$, which can be further converted to $q_{1}$ and $q_{2}$ according to $q_{2} / q_{1}=\tan ^{2} \beta$ and $q_{1}+q_{2}=q$. In practical use with physical inputs, Eqs. (13a) and (13b) are commonly used to evaluate $m_{11}^{2}$ and $m_{22}^{2}$ for given $\tan \beta$ and $v$, together with the quartic couplings determined by

$$
\begin{aligned}
& \lambda_{1}=\frac{M_{h}^{2} \sin ^{2} \alpha+M_{H}^{2} \cos ^{2} \alpha-m_{12}^{2} \tan \beta}{v^{2} \cos ^{2} \beta} \\
& \lambda_{2}=\frac{M_{h}^{2} \cos ^{2} \alpha+M_{H}^{2} \sin ^{2} \alpha-m_{12}^{2} / \tan \beta}{v^{2} \sin ^{2} \beta}, \\
& \lambda_{3}=\frac{1}{v^{2}}\left[\frac{\left(M_{H}^{2}-M_{h}^{2}\right) \sin \alpha \cos \alpha}{\sin \beta \cos \beta}+2 M_{ \pm}^{2}-\frac{m_{12}^{2}}{\sin \beta \cos \beta}\right],
\end{aligned}
$$

$\lambda_{4}=\frac{1}{v^{2}}\left(M_{A}^{2}-2 M_{ \pm}^{2}+\frac{m_{12}^{2}}{\sin \beta \cos \beta}\right)$,

$\lambda_{5}=\frac{1}{v^{2}}\left(\frac{m_{12}^{2}}{\sin \beta \cos \beta}-M_{A}^{2}\right)$.

The potential minima for the Type B, Type C, and Type D cases can be expressed as follows in the physical basis:

$$
\begin{aligned}
& V_{\min , \mathrm{B}}=-\frac{v^{2} \cos ^{2} \beta\left[\left(M_{H}^{2}-M_{h}^{2}\right) \sin \alpha \cos \alpha+\left(M_{H}^{2} \sin ^{2} \alpha+M_{h}^{2} \cos ^{2} \alpha\right) \tan \beta-2 m_{12}^{2}\right]^{2}}{8\left(M_{h}^{2} \cos ^{2} \alpha+M_{H}^{2} \sin ^{2} \alpha-m_{12}^{2} / \tan \beta\right)} \\
& V_{\min , \mathrm{C}}=-\frac{v^{2} \sin ^{2} \beta\left[\left(M_{H}^{2}-M_{h}^{2}\right) \sin \alpha \cos \alpha+\left(M_{H}^{2} \cos ^{2} \alpha+M_{h}^{2} \sin ^{2} \alpha\right) / \tan \beta-2 m_{12}^{2}\right]^{2}}{8\left(M_{H}^{2} \cos ^{2} \alpha+M_{h}^{2} \sin ^{2} \alpha-m_{12}^{2} \tan \beta\right)} \\
& V_{\min , \mathrm{D}}=-\frac{v^{2}}{16}\left[M_{H}^{2}+M_{h}^{2}+\left(M_{H}^{2}-M_{h}^{2}\right) \cos (2 \alpha-2 \beta)\right] .
\end{aligned}
$$


Apparently, the minimal values of the $2 \mathrm{HDM}$ potential in the Type B and Type $\mathrm{C}$ cases are essentially controlled by the input parameters of $\left(M_{h}, M_{H}, m_{12}^{2}, \alpha, \beta\right)$, while the minimal value in the Type $\mathrm{D}$ case is independent of $m_{12}^{2}$.

The GM constraint requires that the Type $\mathrm{D}$ minimum is a GM of the potential in order to protect the corresponding vacuum from decaying to other vacua. To infer whether it is a GM, one can compute all the possible minima listed in Table I and then compare their $V_{\text {min }}$ 's. It is important to mention that the possible minima listed in Table I do not necessarily exist. Table I only provides the possible solutions of the first derivatives vanishing, which should be further checked by the existence conditions in Table I. If $\left(q_{1}, q_{2}, z\right)$ computed for a specific type violates the corresponding existence condition, the solution of this type does not exist. Otherwise, the solution exists. However, this does not necessarily imply it is a minimum since it could also be a maximum or saddle point. Technically, we do not need to check whether the obtained solutions are local minima or other extrema because we are only concerned about the Type $\mathrm{D}$ minimum. As long as $V_{\min }$ of Type $\mathrm{D}$ is lower than the potential values of other existing solutions, Type D must be a GM. Checking the existence of other types of solutions, however, is necessary in this procedure.

Note that the minima summarized in Table I are only for the tree-level potential, while at the loop level they may receive important corrections. To include loop corrections, we use the package Vevacious [82] which is capable of finding the minima of the one-loop effective potential given the minima of the tree-level potential. We will show that loop corrections can change the GM constraint quantitatively but not qualitatively. Therefore the tree-level analytic expressions can be useful tools for understanding the more complicated, loop-corrected GM constraint. Nevertheless, the loop corrections should be included for quantitative studies.

When applying the GM constraint, we shall first impose the BFB [10,83-86] and perturbative unitarity bounds [87-91]. This is because the former is the premise of studying global minima, and the latter avoids too large quartic couplings. Although the unitarity bound is innocuous for the tree-level GM studies, it would drastically enhance the loop corrections. The BFB conditions of the tree-level potential are given as

$$
\begin{aligned}
\lambda_{1,2} & \geq 0, \\
\lambda_{3} & \geq-\sqrt{\lambda_{1} \lambda_{2}}, \\
\lambda_{3}+\lambda_{4}-\left|\lambda_{5}\right| & \geq-\sqrt{\lambda_{1} \lambda_{2}} .
\end{aligned}
$$

However, as recently shown in Ref. [92], the BFB constraints can be alleviated with more feasible parameter regions when the radiative corrections are taken into account. Therefore when studying the loop-level vacua we should check the BFB status of the one-loop effective potential instead of the tree-level potential. In addition, the perturbative unitarity bounds, defined by the requirement that all the scalar scattering amplitudes respect the unitarity condition, are usually considered in the high energy limit of $s \rightarrow \infty$. Recently it has been pointed out in Refs. [89-91] that some amplitudes at finite $s$ might be significantly larger than that in the $s \rightarrow \infty$ limit. Hence, we will adopt the unitarity bounds improved by taking the $s$ dependence into account, which are readily applicable using the SARAH package $[89,93,94]$.

\section{THE GM CONSTRAINTS ON SOME BENCHMARKS}

Given inputs of $\left(M_{h}, M_{H}, M_{A}, M_{ \pm}, m_{12}^{2}, \alpha, \beta\right.$, and $\left.v\right)$ in the physical basis, we can convert them to the potential parameters of $\left(m_{11}^{2}, m_{12}^{2}, m_{22}^{2}\right.$, and $\left.\lambda_{1 \ldots 5}\right)$ in the generic basis and, with the method introduced in Sec. II B, infer whether the corresponding potential violates the GM condition. In this section, we study the GM constraints on the parameters in the physical basis, focusing on two simple yet illustrative scenarios below:

(i) all the heavy Higgs bosons are mass degenerate, i.e., $M_{H}=M_{A}=M_{ \pm}$;

(ii) two of the heavy Higgs bosons are mass degenerate while the remaining are heavier or lighter than the degenerate mass - see Table II. Such a mass spectrum allows exotic decays $[95,96]$.

For scenario (i), we perform a grid scan of $M_{H}=M_{A}=$ $M_{ \pm}$from $200 \mathrm{GeV}$ to $1 \mathrm{TeV}$ at a step of $10 \mathrm{GeV}$, and $m_{12}^{2}$ from 0 to $-(500 \mathrm{GeV})^{2}$ at a step of $(10 \mathrm{GeV})^{2}$. The 2HDM mixing angles $(\alpha, \beta)$ are taken to be consistent with the current LHC constraints on the $125 \mathrm{GeV}$ Higgs boson signal strengths as shown in Fig. 1. For scenario (ii), we summarize the benchmark models in Table II. Taking BP-1, for instance, we perform the grid scan of the heaviest Higgs boson mass $M_{A}$ from $250 \mathrm{GeV}$ to $1 \mathrm{TeV}$ at a step of $10 \mathrm{GeV}$, and the next heavy Higgs boson mass $M_{H}=M_{ \pm}$from $130 \mathrm{GeV}$ up to $M_{A}-100 \mathrm{GeV}$. The soft $\mathbb{Z}_{2}$ breaking parameter $m_{12}^{2}$ still takes the negative values from 0 to $-(500 \mathrm{GeV})^{2}$ at a step of $(10 \mathrm{GeV})^{2}$. In addition, we take $\cos (\beta-\alpha)=0$ (known as the alignment limit) in this case for simplicity. For both scenarios, we set $\tan \beta=1.0$

TABLE II. Summary table of the different benchmark planes (BP) with exotic heavy Higgs boson decays in the 2HDM.

\begin{tabular}{lcc}
\hline \hline & Mass planes & Decays \\
\hline BP-1 & $M_{A}>M_{H}=M_{ \pm}$ & $A \rightarrow\left(H^{ \pm} W^{\mp}, H Z\right)$ \\
BP-2 & $M_{A}<M_{H}=M_{ \pm}$ & $H \rightarrow(A Z, A A), H^{ \pm} \rightarrow A W^{ \pm}$ \\
BP-3 & $M_{H}>M_{A}=M_{ \pm}$ & $H \rightarrow(A Z, A A)$, \\
& & $H \rightarrow\left(H^{+} H^{-}, H^{ \pm} W^{\mp}\right)$ \\
BP-4 & $M_{H}<M_{A}=M_{ \pm}$ & $A \rightarrow H Z, H^{ \pm} \rightarrow H W^{ \pm}$ \\
\hline \hline
\end{tabular}




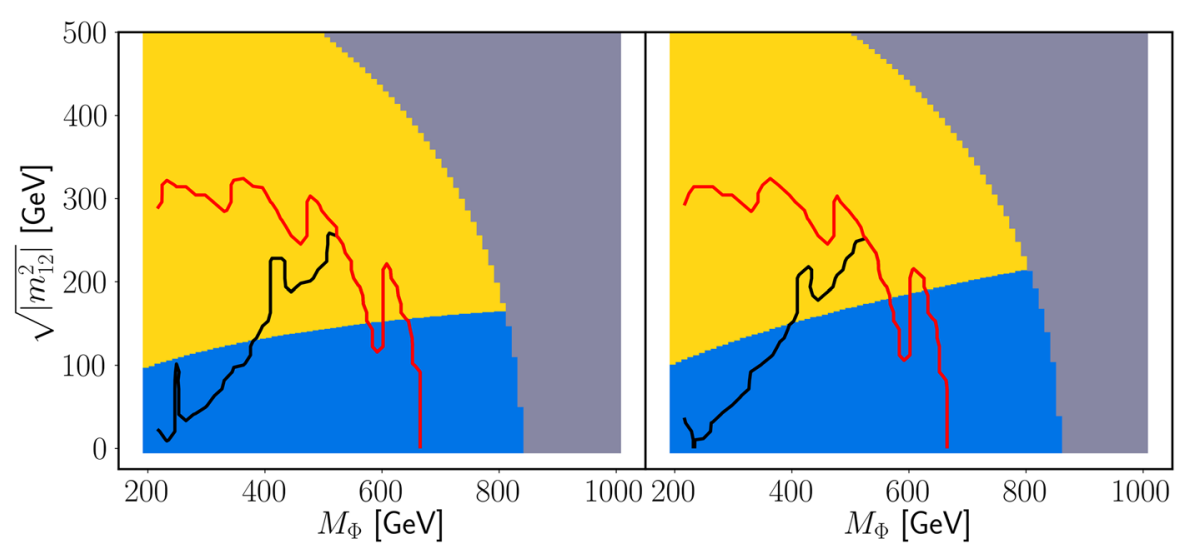

FIG. 2. The GM constraints in the $\left(M_{\Phi}, \sqrt{\left|m_{12}^{2}\right|}\right)$ plane, where $m_{\Phi}$ is defined as the degenerate heavy Higgs boson mass $M_{H}=M_{A}=M_{ \pm} \equiv M_{\Phi}$. The gray regions have already been excluded by the simplistic unitarity and BFB bounds, and the tree-level GM constraints further exclude the yellow regions, leaving only the blue regions allowed by all these constraints. When the loop corrections and the $s$ dependence are included, the unitarity and BFB bounds shift to the red curves, and the GM boundaries shift to the black curves. The left and the right panels assume Type I 2HDM with $\cos (\beta-\alpha)=0.1$ and Type II $2 \mathrm{HDM}$ with $\cos (\beta-\alpha)=0.01$, respectively.

because larger or smaller $\tan \beta$ will be more stringently constrained by the perturbative unitarity bounds.

In Fig. 2, we present the GM constraints for scenario (i), i.e., the mass-degenerate heavy Higgs boson case. All the samples generated in the above way are first filtered by the unitarity and BFB bounds and then constrained by the GM conditions. For comparison, we present results for both a simplistic approach and an improved approach. For the simplistic case, we adopt the conventional unitarity and BFB bounds which exclude the gray regions, and then use
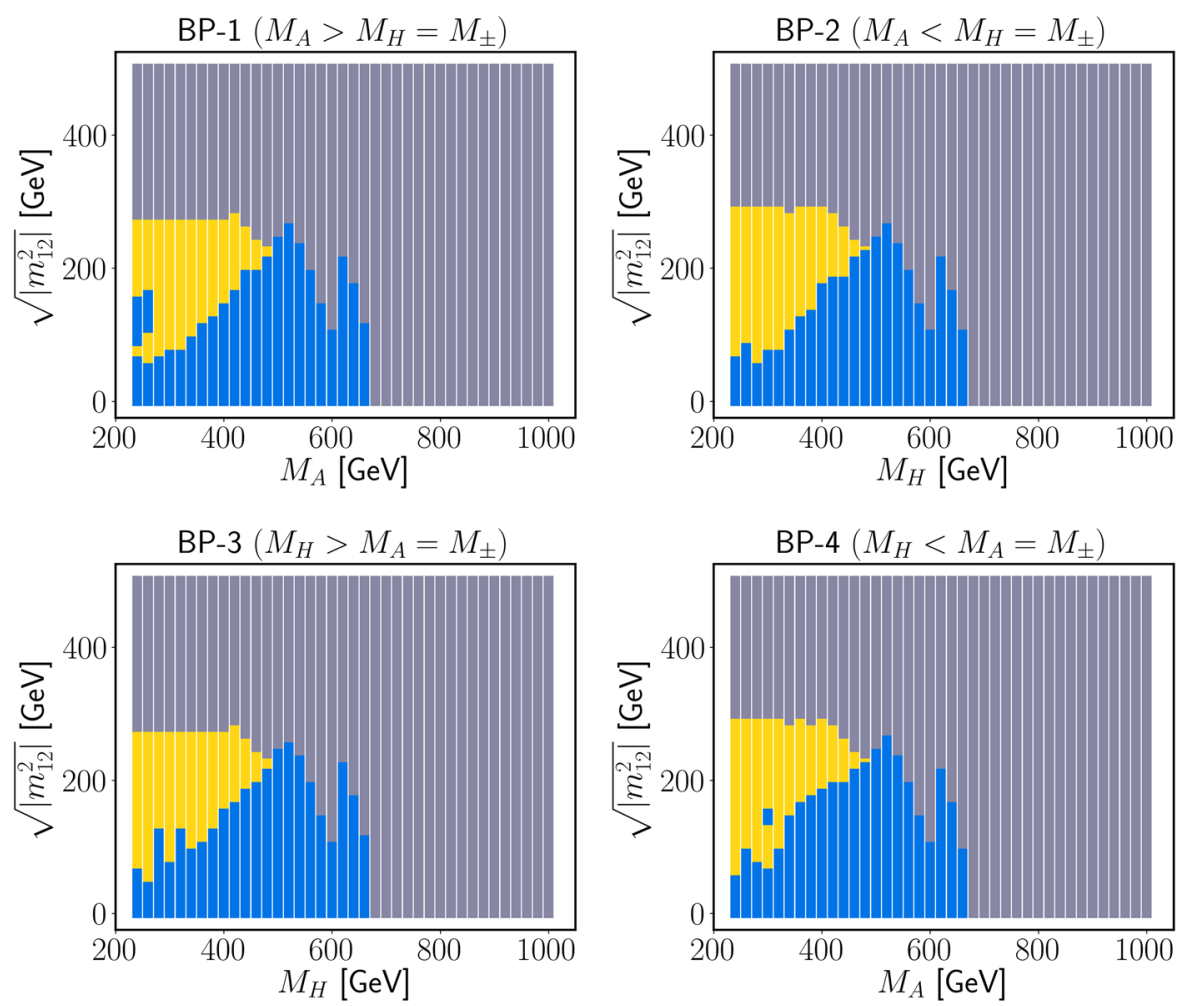

FIG. 3. The GM constraints (excluding yellow) combined with the unitarity and BFB constraints (excluding gray) on some benchmarks tabulated in Table II, with $\cos (\beta-\alpha)=0$ and $\tan \beta=1.0$. The loop corrections and the $s$ dependence have been included. The blue regions are the largest allowed regions by all the constraints in the grid scan-see the text for more details. 
the tree-level GM constraint to further exclude the yellow regions, leaving the blue regions that satisfy all the constraints. The improved case includes the loop corrections and the $s$ dependence (explained at the end of Sec. II B), which change the boundaries of the gray regions to the red curves and the boundaries between the blue and yellow regions to the black curves. As one can see, the loop-corrected GM constraints deviate significantly from the tree-level GM constraints, but both can be violated typically when $\left|m_{12}\right|$ is too large. Due to the significant loop corrections, for the remaining analyses we will adopt the improved constraints while the tree-level constraints are only used to qualitatively understand the results.

In Fig. 3, we present these joint constraints for the heavy Higgs mass spectrum with exotic decays in the $\left(M_{A}, \sqrt{\left|m_{12}^{2}\right|}\right)$ or $\left(M_{H}, \sqrt{\left|m_{12}^{2}\right|}\right)$ plane, with 2HDM mixing angles of $\cos (\beta-\alpha)=0$ and $\tan \beta=1.0$. The allowed regions by the GM constraints should be the same for both Type I and Type II models, provided that the same $2 \mathrm{HDM}$ mixing angles are assumed. The heaviest neutral Higgs boson masses are always labeled as the $x$ axis. The blue regions represent the largest allowed regions by the grid scan of the next heavy Higgs mass in each benchmark model.

\section{THE PHENOMENOLOGY IMPLICATIONS: HEAVY HIGGS BOSON SEARCHES AT THE LHC}

In this section, we will discuss the implications of the GM constraint on the LHC phenomenology of the heavy Higgs boson searches in the general 2HDM. Since we have found that the GM condition is able to further constrain $m_{12}^{2}$, in addition to the unitarity and BFB bounds, the actually allowed ranges of the Higgs boson self-couplings are further restricted. For the cubic Higgs self-couplings in the physical basis, one may check Ref. [97] for details. Accordingly, one can expect that the GM condition will be relevant to the SM-like Higgs boson pair productions and other heavy Higgs search limits at the LHC.

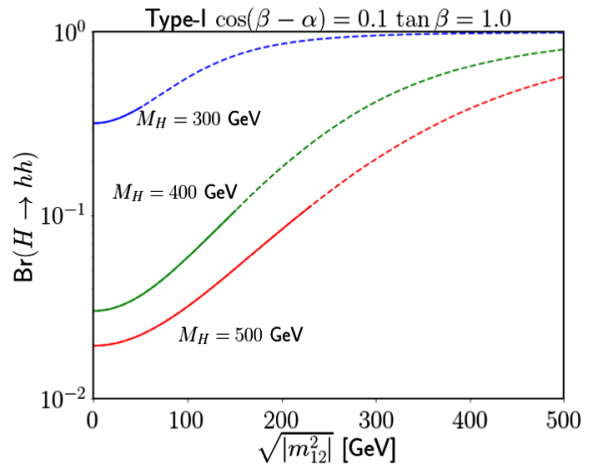

\section{A. The heavy $C P$-even Higgs boson decays into SM-like Higgs boson pairs}

We study the resonance productions of the SM-like Higgs boson pair productions for the degenerate heavy Higgs boson scenario. The exact results for the one-loop Higgs pair production processes at the $p p$ colliders were first studied in Ref. [98]. For the 2HDM case with nonvanishing inputs of $\cos (\beta-\alpha)$, the leading contribution is due to the heavy $C P$-even Higgs boson resonance $H$. In the $m_{12}^{2}<0$ region, we plot the decay branching fraction of $\operatorname{Br}[H \rightarrow h h]$ for the Type I model [with parameters of $\cos (\beta-\alpha)=0.01$ and $\tan \beta=1.0]$ and the Type II model (with parameters of $\cos (\beta-\alpha)=0.1$ and $\tan \beta=1.0$ ) in Fig. 4, for three different inputs of the heavy $C P$-even Higgs boson masses. The decay branching fractions of $\operatorname{Br}[H \rightarrow h h]$ are apparently suppressed in the Type II model, with a small $\cos (\beta-\alpha)$ input, as compared to the Type I model. With the GM condition, the allowed ranges of $\sqrt{\left|m_{12}^{2}\right|}$ are further restricted, which were also displayed in Fig. 2 previously.

We obtain the heavy $C P$-even Higgs boson production cross sections at the LHC $13 \mathrm{TeV}$ runs, by using the SUSHI package [99]. For the parton distributions, we use NNPDF. Both the heavy resonance searches for $H \rightarrow h h \rightarrow b \bar{b} \gamma \gamma$ [100] and $H \rightarrow h h \rightarrow b \bar{b} b \bar{b}$ [101] were taken into account. In Fig. 5, the current LHC $13 \mathrm{TeV}$ search limits on the SM-like Higgs boson pairs via these two channels, as well as the theoretically allowed regions, are presented in the $\left(M_{H}, \sqrt{\left|m_{12}^{2}\right|}\right)$ plane. For the Type I model, the current LHC search limits have excluded the heavy Higgs boson mass ranges of $250 \mathrm{GeV} \lesssim M_{H} \lesssim 350 \mathrm{GeV}$. Meanwhile, the search limits on the Type II model are much smaller in the theoretically allowed region, since the corresponding alignment parameter of $\cos (\beta-\alpha)$ was suppressed from the LHC signal strengths. For both Type I and Type II, most of the LHC excluded regions are actually out of the theoretically allowed regions, showing the robustness of the combinations of GM constraints and other theoretical bounds in the $H \rightarrow h h$ process.

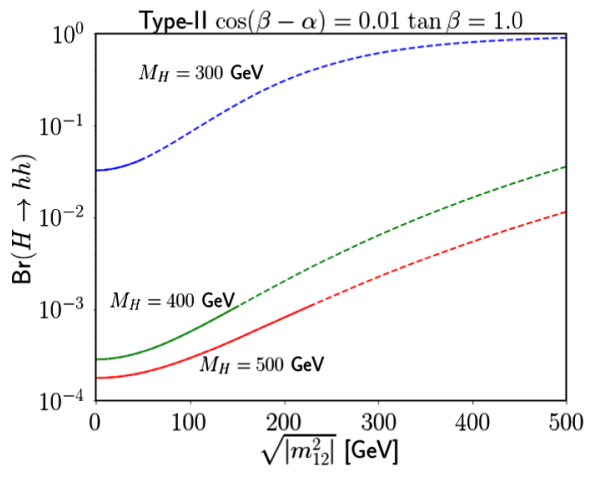

FIG. 4. The decay branching fractions of $\operatorname{Br}[H \rightarrow h h]$, for the Type I model (left panel) and Type II model (right panel). The solid or dashed curves represent the parameter regions that are allowed or excluded by the GM conditions, respectively. The loop corrections and the $s$ dependence have been included. 

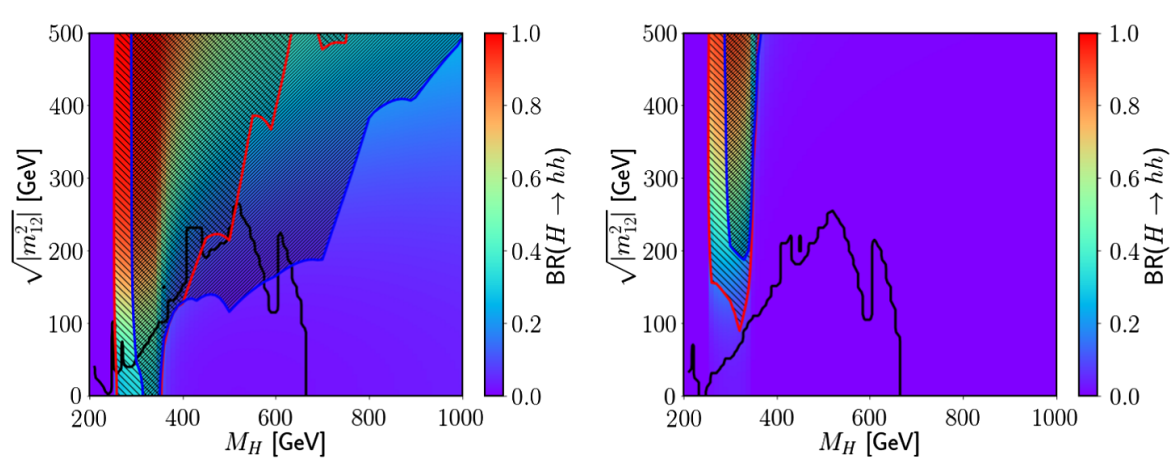

FIG. 5. The current LHC $13 \mathrm{TeV}$ search limits on the resonance productions of SM-like Higgs boson pairs, for the Type I model (left panel) and Type II model (right panel). The red and blue hatched regions have been excluded by the $h h \rightarrow b \bar{b} \gamma \gamma$ and $h h \rightarrow b \bar{b} b \bar{b}$, respectively. The black contours represent the theoretically (including the GM and unitarity and BFB constraints) allowed regions.

\section{B. The exotic heavy Higgs boson decays}

In general, the cubic self-couplings control the partial decay widths of heavy Higgs bosons, such as $H \rightarrow h h, A A$, $H^{+} H^{-}$, and so on. The constraints from the GM requirements turn out to be relevant to these partial decay widths, and hence to all possible decay branching fractions of heavy Higgs bosons. The possible exotic heavy Higgs boson decay modes were previously tabulated in Table II in the alignment limit.

Currently, the most recent LHC search limits on a heavy Higgs boson decaying into a $Z$ boson plus another heavy Higgs boson via the $\ell^{+} \ell^{-} b \bar{b}$ final state can be found in Ref. [102]. The experimental search limits of $\sigma \times \operatorname{Br}[A \rightarrow Z H] \times \operatorname{Br}[H \rightarrow b \bar{b}]$ have been projected to the two-dimensional plane of $\left(M_{H}, M_{A}\right)$ by assuming that $M_{A}>M_{H}$. Such exotic heavy Higgs decay searches have also been studied in Ref. [96] via different final states of $2 b+4 \ell$ or $4 b+2 \ell$. Here, we use the observed upper limits on both $g g \rightarrow A \rightarrow Z H$ and $g g \rightarrow H \rightarrow Z A$ processes by assuming that the search limits are insensitive to the parity properties of the heavy Higgs bosons. Through Figs. 6-9, we present the current LHC $13 \mathrm{TeV}$ search limits on the exotic heavy Higgs boson decay modes in the $\left(M_{A}, M_{H}\right)$ plane (for BP-1 and BP-4) or the $\left(M_{H}, M_{A}\right)$ plane (for BP-2 and BP-3). In Ref. [102], the mass ranges of the experimental searches were taken to be $M_{A} \in$ $(230,800) \mathrm{GeV}$ and $M_{H} \in(130,700) \mathrm{GeV}$. For all four benchmark planes, the current LHC $13 \mathrm{TeV}$ experimental search limits have excluded the regions where the nextheaviest Higgs boson masses are $\lesssim 300 \mathrm{GeV}$.

As shown in Figs. 6-9, for all the cases presented here, the LHC excluded regions partially overlap with theoretically allowed regions (marked by the black contour), which implies that the GM constraints are complementary to the LHC constraints. When $m_{12}^{2}$ changes from zero (left panels) to negative values (right panels), the theoretically allowed regions shrink, leading to preferences for smaller branching ratios. Generally speaking, more negative $m_{12}^{2}$ is more stringently constrained by the GM constraints, which is consistent with what has been shown in Figs. 2 and 3. This can be understood by the analytical expressions of potential minima in Eqs. (15), which can be reduced to

$$
V_{\min , \mathrm{B}} \rightarrow-\frac{\left(M_{h}^{2} \tan \beta-2 m_{12}^{2}\right)^{2} v^{2} \cos ^{2} \beta}{8\left(M_{h}^{2} \sin ^{2} \beta+M_{H}^{2} \cos ^{2} \beta-m_{12}^{2} / \tan \beta\right)},
$$

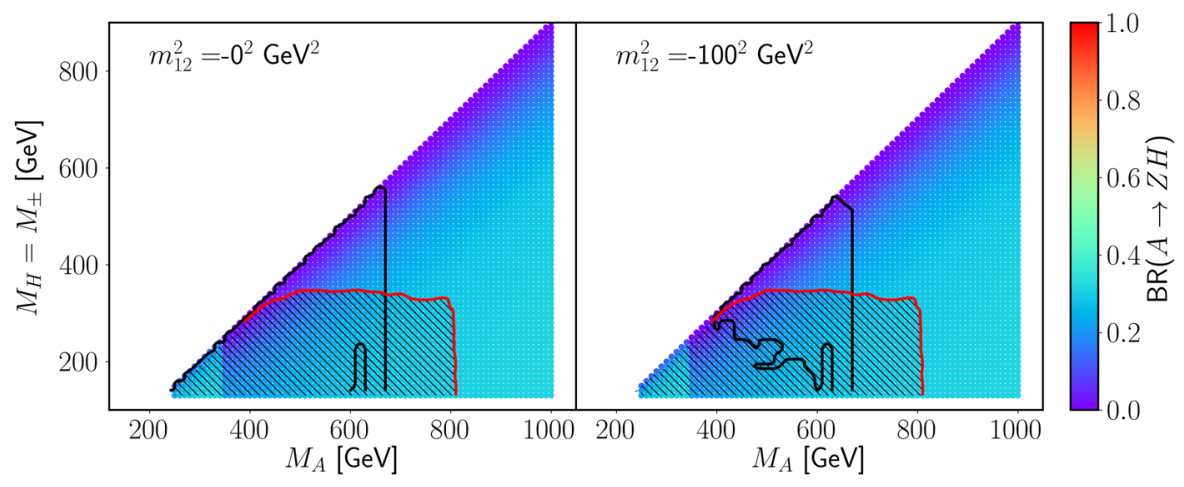

FIG. 6. The current LHC $13 \mathrm{TeV}$ search limits on the $A \rightarrow Z H$ in the BP-1 case with $\cos (\beta-\alpha)=0$ and tan $\beta=1.0$ input. The red hatched regions have been excluded by the LHC $13 \mathrm{TeV}$ searches for $A \rightarrow Z H$. The black contours represent the theoretically (including the GM and unitarity and BFB constraints) allowed regions. 


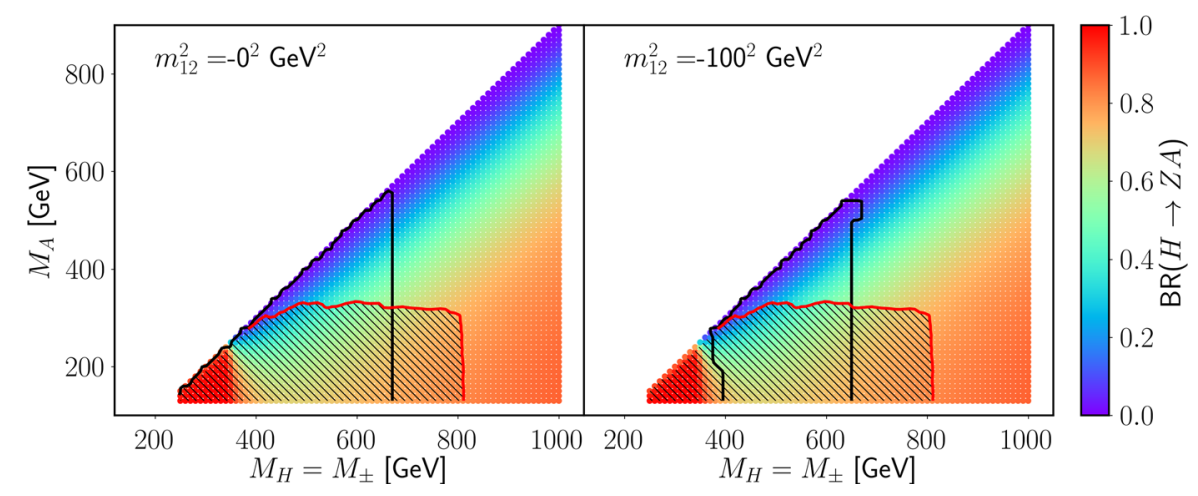

FIG. 7. Similar to Fig. 6, but for the LHC $13 \mathrm{TeV}$ search limits on $H \rightarrow Z A$ in the BP-2 case.

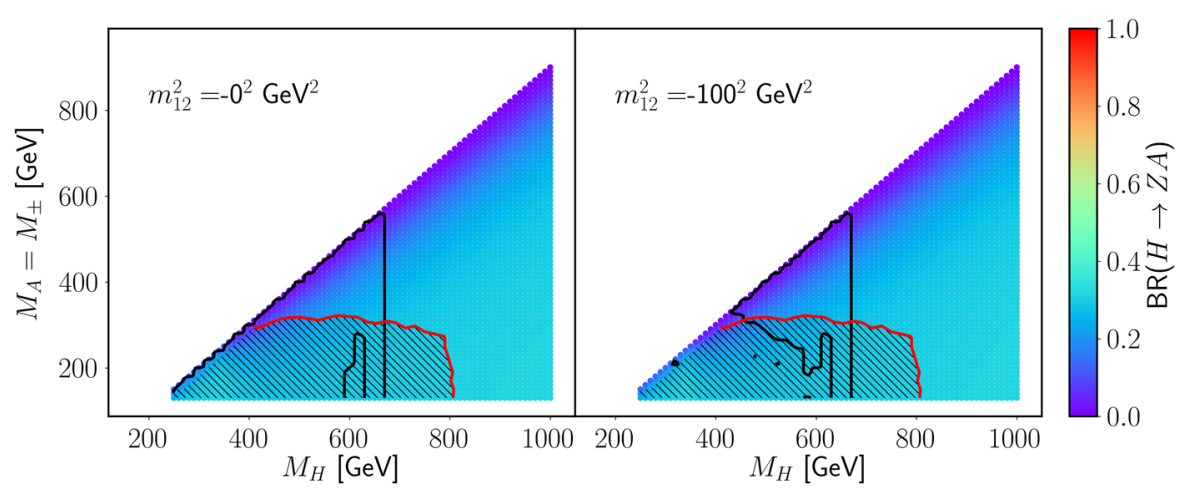

FIG. 8. Similar to Fig. 6, but for the LHC $13 \mathrm{TeV}$ search limits on $H \rightarrow Z A$ in the BP-3 case.

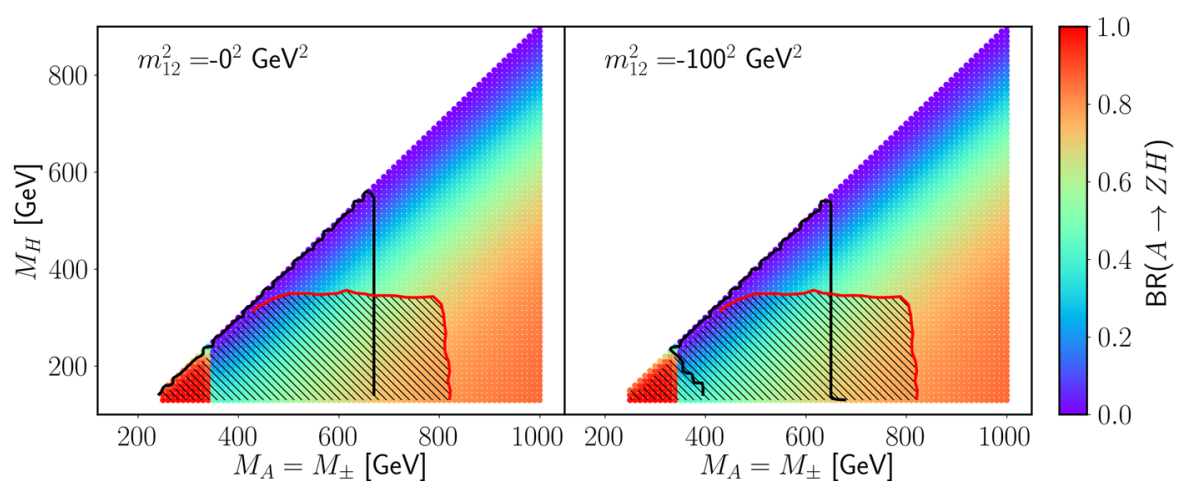

FIG. 9. Similar to Fig. 6, but for the LHC $13 \mathrm{TeV}$ search limits on $A \rightarrow Z H$ in the BP-4 case.

$V_{\min , \mathrm{C}} \rightarrow-\frac{\left(M_{h}^{2} / \tan \beta-2 m_{12}^{2}\right)^{2} v^{2} \sin ^{2} \beta}{8\left(M_{H}^{2} \sin ^{2} \beta+M_{h}^{2} \cos ^{2} \beta-m_{12}^{2} \tan \beta\right)}$,

$V_{\min , \mathrm{D}} \rightarrow-\frac{v^{2}}{8} M_{h}^{2}$

in the alignment limit of $\cos (\beta-\alpha)=0$. To have both conditions of $V_{\min , \mathrm{D}}<V_{\min , \mathrm{B}}$ and $V_{\min , \mathrm{D}}<V_{\min , \mathrm{C}}$ hold with a more negative input of $m_{12}^{2}$, one thus demands a larger input of $M_{H}$.

For the BP-1 and BP-4 cases, a more negative input of $m_{12}^{2}$ pushes $M_{A}$ and $M_{H}$ closer to each other. Therefore, one can expect the current experimental searches via the
$H \rightarrow Z A$ mode to become more challenging, since the transverse momenta of final-state $b$ jets and leptons are smaller. Similar situations can be envisioned for the BP-2 and BP-3 cases as well, but for the different decay mode of $A \rightarrow Z H$. This suggests that the heavy Higgs boson spectrum involving exotic decay modes may be hidden from the LHC experimental searches, with negative inputs of $m_{12}^{2}$ and the GM constraint taken into account.

\section{CONCLUSION}

In the scalar potential of the general $2 \mathrm{HDM}$, it is likely that several minima may coexist. The usually considered 
vacuum can thus become a local minimum, and it may decay into a deeper one. To avoid this vacuum instability at the tree level, we impose the GM condition to the 2HDM potential.

According to our analysis, it turns out that the GM condition can impose a more stringent bound on the $m_{12}^{2}$ parameter when it is in the negative region. Besides, we find that large or small inputs of $\tan \beta$ can impose stringent bounds on the heavy Higgs boson masses for the illustrated cases. Hence, we focus on the parameter input of $\tan \beta=$ 1.0 in our discussion. Two different scenarios in the heavy Higgs boson sector were considered in our analysis. For the mass-degenerate heavy Higgs bosons, we find that the actually expected decay branching fractions of $\operatorname{Br}[H \rightarrow h h]$ with a nonvanishing alignment parameter are restricted into smaller ranges. The current LHC $13 \mathrm{TeV}$ searches for SM-like Higgs boson pairs via $b \bar{b} \gamma \gamma$ and $b \bar{b} b \bar{b}$ final states are more sensitive in the Type I benchmark model, compared to the Type II benchmark model with a suppressed $\cos (\beta-\alpha)$ input. For the heavy Higgs boson spectrum involving exotic decays, the GM constraint can put more stringent bounds with more negative inputs of $m_{12}^{2}$. The current LHC $13 \mathrm{TeV}$ run has performed searches for the heavy Higgs boson with an exotic decay mode of $A \rightarrow Z H \rightarrow \ell^{+} \ell^{-} b \bar{b}$. We projected the experimental search limits on the $\left(M_{A}, M_{H}\right)$ plane (when $M_{A}>M_{H}$ ) or the $\left(M_{H}, M_{A}\right)$ plane (when $\left.M_{H}>M_{A}\right)$. We also note that the GM constraint can squeeze the heavy $C P$-even Higgs boson mass $M_{H}$ into larger values with the negative inputs of $m_{12}^{2}$. Consequently, such parameter regions bring difficulty for the future LHC searches via the exotic heavy Higgs boson decay channels.

\section{ACKNOWLEDGMENTS}

The work of N. C. is supported by the National Natural Science Foundation of China (under Grant No. 11575176) and Center for Future High Energy Physics (CFHEP). The work of Y.C. W. is partially supported by the Natural Sciences and Engineering Research Council of Canada. N.C. thanks Center for High Energy Physics Peking University for their hospitality when part of this work was prepared.
[1] G. C. Branco, P. M. Ferreira, L. Lavoura, M. N. Rebelo, M. Sher, and J. P. Silva, Theory and phenomenology of twoHiggs-doublet models, Phys. Rep. 516, 1 (2012).

[2] H. E. Haber and G. L. Kane, The search for supersymmetry: Probing physics beyond the standard model, Phys. Rep. 117, 75 (1985).

[3] A. Djouadi, The anatomy of electro-weak symmetry breaking. II. The Higgs bosons in the minimal supersymmetric model, Phys. Rep. 459, 1 (2008).

[4] T. D. Lee, A theory of spontaneous T violation, Phys. Rev. D 8, 1226 (1973).

[5] J.E. Kim, Light Pseudoscalars, particle physics and cosmology, Phys. Rep. 150, 1 (1987).

[6] P. M. Ferreira, R. Santos, and A. Barroso, Stability of the tree-level vacuum in two Higgs doublet models against charge or CP spontaneous violation, Phys. Lett. B 603, 219 (2004); Erratum, Phys. Lett. B 629, 114(E) (2005).

[7] A. Barroso, P. M. Ferreira, and R. Santos, Charge and $C P$ symmetry breaking in two Higgs doublet models, Phys. Lett. B 632, 684 (2006).

[8] A. Barroso, P. M. Ferreira, and R. Santos, Some remarks on tree-level vacuum stability in two Higgs doublet models, Afr. J. Math. Phys. 3, 103 (2006).

[9] A. Barroso, P. M. Ferreira, and R. Santos, Tree-level vacuum stability in multi Higgs models, Proc. Sci. HEP2005 (2006) 337.

[10] I. P. Ivanov, Minkowski space structure of the Higgs potential in 2HDM, Phys. Rev. D 75, 035001 (2007); Erratum, Phys. Rev. D 76, 039902(E) (2007).
[11] A. Barroso, P. M. Ferreira, R. Santos, and J. P. Silva, Stability of the normal vacuum in multi-Higgs-doublet models, Phys. Rev. D 74, 085016 (2006).

[12] I. P. Ivanov, Minkowski space structure of the Higgs potential in 2HDM. II. Minima, symmetries, and topology, Phys. Rev. D 77 (2008) 015017.

[13] A. Barroso, P. M. Ferreira, and R. Santos, Neutral minima in two-Higgs doublet models, Phys. Lett. B 652, 181 (2007).

[14] I. P. Ivanov, Can 2HDM support fermion-stabilized bubbles of false vacuum? arXiv:0706.4332.

[15] I. P. Ivanov, Thermal evolution of the ground state of the most general 2HDM, Acta Phys. Pol. B 40, 2789 (2009).

[16] I. P. Ivanov and C. C. Nishi, Properties of the general NHDM. I. The Orbit space, Phys. Rev. D 82, 015014 (2010).

[17] N. Barros e Sa, A. Barroso, P. Ferreira, and R. Santos, Vacuum Stability in two-Higgs doublet models, Proc. Sci., CHARGED2008 (2008) 014.

[18] I. F. Ginzburg, I. P. Ivanov, and K. A. Kanishev, The evolution of vacuum states and phase transitions in 2HDM during cooling of Universe, Phys. Rev. D 81, 085031 (2010).

[19] I. P. Ivanov, Properties of the general NHDM. II. Higgs potential and its symmetries, J. High Energy Phys. 07 (2010) 020.

[20] R. A. Battye, G. D. Brawn, and A. Pilaftsis, Vacuum topology of the two Higgs doublet model, J. High Energy Phys. 08 (2011) 020. 
[21] A. Barroso, P. M. Ferreira, I. P. Ivanov, R. Santos, and J. P. Silva, Evading death by vacuum, Eur. Phys. J. C 73, 2537 (2013).

[22] A. Barroso, P. M. Ferreira, I. Ivanov, R. Santos, and J. P. Silva, Avoiding death by vacuum, J. Phys. Conf. Ser. 447, 012051 (2013).

[23] A. Barroso, P. M. Ferreira, I. P. Ivanov, and R. Santos, Metastability bounds on the two Higgs doublet model, J. High Energy Phys. 06 (2013) 045.

[24] A. Barroso, P. M. Ferreira, I. Ivanov, and R. Santos, Treelevel metastability bounds in two-Higgs doublet models, in Proceedings, 1st Toyama International Workshop on Higgs as a Probe of New Physics 2013 (HPNP2013): Toyama, Japan, 2013 (2013).

[25] I. P. Ivanov and J. P. Silva, Tree-level metastability bounds for the most general two Higgs doublet model, Phys. Rev. D 92, 055017 (2015).

[26] S. R. Coleman, The fate of the false vacuum. 1. Semiclassical theory, Phys. Rev. D 15, 2929 (1977); Erratum, Phys. Rev. D 16, 1248(E) (1977).

[27] C. G. Callan, Jr. and S. R. Coleman, The fate of the false vacuum. 2. First quantum corrections, Phys. Rev. D 16, 1762 (1977).

[28] X.-J. Xu, Tree-level vacuum stability of two-Higgs-doublet models and new constraints on the scalar potential, Phys. Rev. D 95, 115019 (2017).

[29] K. Hartling, K. Kumar, and H. E. Logan, The decoupling limit in the Georgi-Machacek model, Phys. Rev. D 90, 015007 (2014).

[30] X.-J. Xu, Minima of the scalar potential in the type II seesaw model: From local to global, Phys. Rev. D 94, 115025 (2016).

[31] P. S. Bhupal Dev, R. N. Mohapatra, W. Rodejohann, and $\mathrm{X}$.-J. $\mathrm{Xu}$, Vacuum structure of the left-right symmetric model, arXiv:1811.06869.

[32] J. Baglio, A. Djouadi, R. Gröber, M. M. Mühlleitner, J. Quevillon, and M. Spira, The measurement of the Higgs self-coupling at the LHC: theoretical status, J. High Energy Phys. 04 (2013) 151.

[33] D. Y. Shao, C.S. Li, H. T. Li, and J. Wang, Threshold resummation effects in Higgs boson pair production at the LHC, J. High Energy Phys. 07 (2013) 169.

[34] N. Chen, C. Du, Y. Fang, and L.-C. Lü, LHC searches for the heavy Higgs boson via two B jets plus Diphoton, Phys. Rev. D 89, 115006 (2014).

[35] C.-R. Chen and I. Low, Double take on new physics in double Higgs boson production, Phys. Rev. D 90, 013018 (2014).

[36] V. Barger, L. L. Everett, C. B. Jackson, A. D. Peterson, and G. Shaughnessy, Measuring the two-Higgs doublet model scalar potential at LHC14, Phys. Rev. D 90, 095006 (2014).

[37] L. Bian and N. Chen, Higgs pair productions in the $C P$-violating two-Higgs-doublet model, J. High Energy Phys. 09 (2016) 069.

[38] Q.-H. Cao, G. Li, B. Yan, D.-M. Zhang, and H. Zhang, Double Higgs production at the $14 \mathrm{TeV}$ LHC and a $100 \mathrm{TeV} p p$ collider, Phys. Rev. D 96, 095031 (2017).

[39] W. Kilian, S. Sun, Q.-S. Yan, X. Zhao, and Z. Zhao, New physics in multi-Higgs boson final states, J. High Energy Phys. 06 (2017) 145.
[40] G. Cacciapaglia, H. Cai, A. Carvalho, A. Deandrea, T. Flacke, B. Fuks, D. Majumder, and H.-S. Shao, Probing vector-like quark models with Higgs-boson pair production, J. High Energy Phys. 07 (2017) 005.

[41] L. Di Luzio, R. Gröber, and M. Spannowsky, Maxi-sizing the trilinear Higgs self-coupling: How large could it be?, Eur. Phys. J. C 77, 788 (2017).

[42] A. Alves, T. Ghosh, and K. Sinha, Can we discover double Higgs production at the LHC?, Phys. Rev. D 96, 035022 (2017).

[43] T. Corbett, A. Joglekar, H.-L. Li, and J.-H. Yu, Exploring extended scalar sectors with Di-Higgs signals: A Higgs EFT perspective, J. High Energy Phys. 05 (2018) 061.

[44] R. Grober, M. Muhlleitner, and M. Spira, Higgs pair production at NLO QCD for $C P$-violating Higgs sectors, Nucl. Phys. B925, 1 (2017).

[45] J. Ren, R.-Q. Xiao, M. Zhou, Y. Fang, H.-J. He, and W. Yao, LHC search of new Higgs boson via resonant DiHiggs production with decays into 4W, J. High Energy Phys. 06 (2018) 090.

[46] P. Basler, M. Mühlleitner, and J. Wittbrodt, The $C P$-violating $2 \mathrm{HDM}$ in light of a strong first order electroweak phase transition and implications for Higgs pair production, J. High Energy Phys. 03 (2018) 061.

[47] S. Dawson and M. Sullivan, Enhanced di-Higgs production in the complex Higgs singlet model, Phys. Rev. D 97, 015022 (2018).

[48] A. Adhikary, S. Banerjee, R. K. Barman, B. Bhattacherjee, and S. Niyogi, Revisiting the non-resonant Higgs pair production at the HL-LHC, J. High Energy Phys. 07 (2018) 116.

[49] D. Gonçalves, T. Han, F. Kling, T. Plehn, and M. Takeuchi, Higgs boson pair production at future hadron colliders: From kinematics to dynamics, Phys. Rev. D 97, 113004 (2018).

[50] G. Aad et al. (ATLAS Collaboration), Measurement of Higgs boson production in the diphoton decay channel in pp collisions at center-of-mass energies of 7 and $8 \mathrm{TeV}$ with the ATLAS detector, Phys. Rev. D 90, 112015 (2014).

[51] G. Aad et al. (ATLAS Collaboration), Measurements of Higgs boson production and couplings in the four-lepton channel in pp collisions at center-of-mass energies of 7 and $8 \mathrm{TeV}$ with the ATLAS detector, Phys. Rev. D 91, 012006 (2015).

[52] G. Aad et al. (ATLAS Collaboration), Observation and measurement of Higgs boson decays to $\mathrm{WW}^{*}$ with the ATLAS detector, Phys. Rev. D 92, 012006 (2015).

[53] G. Aad et al. (ATLAS Collaboration), Search for the Standard Model Higgs boson produced in association with top quarks and decaying into $b \bar{b}$ in pp collisions at $\sqrt{s}=$ $8 \mathrm{TeV}$ with the ATLAS detector, Eur. Phys. J. C 75, 349 (2015).

[54] G. Aad et al. (ATLAS Collaboration), Search for the $b \bar{b}$ decay of the Standard Model Higgs boson in associated $(W / Z) H$ production with the ATLAS detector, J. High Energy Phys. 01 (2015) 069.

[55] G. Aad et al. (ATLAS Collaboration), Evidence for the Higgs-boson Yukawa coupling to tau leptons with the ATLAS detector, J. High Energy Phys. 04 (2015) 117. 
[56] V. Khachatryan et al. (CMS Collaboration), Observation of the diphoton decay of the Higgs boson and measurement of its properties, Eur. Phys. J. C 74, 3076 (2014).

[57] S. Chatrchyan et al. (CMS Collaboration), Measurement of the properties of a Higgs boson in the four-lepton final state, Phys. Rev. D 89, 092007 (2014).

[58] S. Chatrchyan et al. (CMS Collaboration), Measurement of Higgs boson production and properties in the WW decay channel with leptonic final states, J. High Energy Phys. 01 (2014) 096.

[59] V. Khachatryan et al. (CMS Collaboration), Search for a Standard Model Higgs boson produced in association with a top-quark pair and decaying to bottom quarks using a matrix element method, Eur. Phys. J. C 75, 251 (2015).

[60] S. Chatrchyan et al. (CMS Collaboration), Search for the standard model Higgs boson produced in association with a $\mathrm{W}$ or a $\mathrm{Z}$ boson and decaying to bottom quarks, Phys. Rev. D 89, 012003 (2014).

[61] S. Chatrchyan et al. (CMS Collaboration), Evidence for the $125 \mathrm{GeV}$ Higgs boson decaying to a pair of $\tau$ leptons, J. High Energy Phys. 05 (2014) 104.

[62] ATLAS Collaboration, Measurements of Higgs boson properties in the diphoton decay channel with $36.1 \mathrm{fb}^{-1}$ $p p$ collision data at the center-of-mass energy of $13 \mathrm{TeV}$ with the ATLAS detector, CERN, Technical Report No. ATLAS-CONF-2017-045, 2017.

[63] ATLAS Collaboration, Combined measurements of Higgs boson production and decay in the $H \rightarrow Z Z^{*} \rightarrow 4 \ell$ and $H \rightarrow \gamma \gamma$ channels using $\sqrt{s}=13 \mathrm{TeV}$ pp collision data collected with the ATLAS experiment, CERN, Technical Report No. ATLAS-CONF-2017-047, 2017.

[64] ATLAS Collaboration, Measurement of the Higgs boson coupling properties in the $H \rightarrow Z Z^{*} \rightarrow 4 \ell$ decay channel at $\sqrt{s}=13 \mathrm{TeV}$ with the ATLAS detector, CERN, Technical Report No. ATLAS-CONF-2017-043, 2017.

[65] ATLAS Collaboration, Measurements of the Higgs boson production cross section via Vector Boson Fusion and associated $W H$ production in the $W W^{*} \rightarrow \ell \nu \ell \nu$ decay mode with the ATLAS detector at $\sqrt{s}=13 \mathrm{TeV}, \mathrm{CERN}$, Technical Report No. ATLAS-CONF-2016-112, 2016.

[66] M. Aaboud et al. (ATLAS Collaboration), Evidence for the $H \rightarrow b \bar{b}$ decay with the ATLAS detector, J. High Energy Phys. 12 (2017) 024.

[67] M. D. Campos, D. Cogollo, M. Lindner, T. Melo, F. S. Queiroz, and W. Rodejohann, Neutrino masses and absence of flavor changing interactions in the 2HDM from Gauge principles, J. High Energy Phys. 08 (2017) 092.

[68] ATLAS Collaboration, Search for the Standard Model Higgs boson produced in association with top quarks and decaying into $b \bar{b}$ in $p p$ collisions at $\sqrt{s}=13 \mathrm{TeV}$ with the ATLAS detector, CERN, Technical Report No. ATLASCONF-2016-080, 2016.

[69] M. Aaboud et al. (ATLAS Collaboration), Search for the Dimuon Decay of the Higgs Boson in $p p$ Collisions at $\sqrt{s}=13 \mathrm{TeV}$ with the ATLAS Detector, Phys. Rev. Lett. 119, 051802 (2017).

[70] CMS Collaboration, Measurements of properties of the Higgs boson decaying into four leptons in pp collisions at $\sqrt{s}=13 \mathrm{TeV}$, CERN, Technical Report No. CMS-PASHIG-16-041, 2017.
[71] A. M. Sirunyan et al. (CMS Collaboration), Measurements of properties of the Higgs boson decaying into the fourlepton final state in $\mathrm{pp}$ collisions at $\sqrt{(} s)=13 \mathrm{TeV}$, J. High Energy Phys. 11 (2017) 047.

[72] CMS Collaboration, Higgs to WW measurements with $15.2 \mathrm{fb}^{-1}$ of $13 \mathrm{TeV}$ proton-proton collisions, CERN, Technical Report No. CMS-PAS-HIG-16-021, 2017.

[73] A. M. Sirunyan et al. (CMS Collaboration), Inclusive Search for a Highly Boosted Higgs Boson Decaying to a Bottom Quark-Antiquark Pair, Phys. Rev. Lett. 120, 071802 (2018).

[74] A. M. Sirunyan et al. (CMS Collaboration), Observation of the Higgs boson decay to a pair of tau leptons, Phys. Lett. B 779, 283 (2018).

[75] CMS Collaboration, Search for the associated production of a Higgs boson with a top quark pair in final states with a $\tau$ lepton at $\sqrt{s}=13 \mathrm{TeV}, \mathrm{CERN}$, Technical Report No. CMS-PAS-HIG-17-003, 2017.

[76] G. Aad et al. (ATLAS and CMS Collaborations), Measurements of the Higgs boson production and decay rates and constraints on its couplings from a combined ATLAS and CMS analysis of the LHC pp collision data at $\sqrt{s}=7$ and 8 TeV, J. High Energy Phys. 08 (2016) 045,

[77] ATLAS Collaboration, Combined measurements of Higgs boson production and decay using up to $80 \mathrm{fb}^{-1}$ of protonproton collision data at $\sqrt{s}=13 \mathrm{TeV}$ collected with the ATLAS experiment, CERN, Technical Report No. ATLASCONF-2018-031, 2018.

[78] A. M. Sirunyan et al. (CMS Collaboration), Combined measurements of Higgs boson couplings in proton-proton collisions at $\sqrt{s}=13 \mathrm{TeV}$, arXiv:1809.10733.

[79] J. Gu, H. Li, Z. Liu, S. Su, and W. Su, Learning from Higgs physics at future Higgs factories, J. High Energy Phys. 12 (2017) 153,

[80] P. M. Ferreira, J. F. Gunion, H. E. Haber, and R. Santos, Probing wrong-sign Yukawa couplings at the LHC and a future linear collider, Phys. Rev. D 89, 115003 (2014).

[81] L. Wang, R. Shi, and X.-F. Han, Wrong sign Yukawa coupling of the $2 \mathrm{HDM}$ with a singlet scalar as dark matter confronted with dark matter and Higgs data, Phys. Rev. D 96, 115025 (2017).

[82] J. E. Camargo-Molina, B. O'Leary, W. Porod, and F. Staub, Vevacious: A tool for finding the global minima of one-loop effective potentials with many scalars, Eur. Phys. J. C 73, 2588 (2013).

[83] M. Maniatis, A. von Manteuffel, O. Nachtmann, and F. Nagel, Stability and symmetry breaking in the general twoHiggs-doublet model, Eur. Phys. J. C 48, 805 (2006).

[84] C. C. Nishi, The structure of potentials with N Higgs doublets, Phys. Rev. D 76, 055013 (2007).

[85] P. M. Ferreira and D. R. T. Jones, Bounds on scalar masses in two Higgs doublet models, J. High Energy Phys. 08 (2009) 069.

[86] I. P. Ivanov, M. Köpke, and M. Mühlleitner, Algorithmic Boundedness-From-Below conditions for generic scalar potentials, Eur. Phys. J. C 78, 413 (2018).

[87] A. Arhrib, Unitarity constraints on scalar parameters of the standard and two Higgs doublets model, in Workshop on Noncommutative Geometry, Superstrings and Particle Physics Rabat, Morocco, 2000 (2000). 
[88] S. Kanemura and K. Yagyu, Unitarity bound in the most general two Higgs doublet model, Phys. Lett. B 751, 289 (2015).

[89] M. D. Goodsell and F. Staub, Unitarity constraints on general scalar couplings with SARAH, Eur. Phys. J. C 78, 649 (2018).

[90] M. D. Goodsell and F. Staub, Improved unitarity constraints in Two-Higgs-Doublet-Models, Phys. Lett. B 788, 206 (2019).

[91] M. E. Krauss and F. Staub, Unitarity constraints in triplet extensions beyond the large s limit, Phys. Rev. D 98, 015041 (2018).

[92] F. Staub, Reopen parameter regions in Two-Higgs Doublet Models, Phys. Lett. B 776, 407 (2018).

[93] F. Staub Sarah, arXiv:0806.0538.

[94] F. Staub, SARAH 4: A tool for (not only SUSY) model builders, Comput. Phys. Commun. 185, 1773 (2014).

[95] F. Kling, J. M. No, and S. Su, Anatomy of exotic Higgs decays in 2HDM, J. High Energy Phys. 09 (2016) 093.

[96] B. Coleppa, B. Fuks, P. Poulose, and S. Sahoo, Seeking heavy Higgs bosons through cascade decays, Phys. Rev. D 97, 075007 (2018).

[97] D. Lopez-Val and J. Sola, Neutral Higgs-pair production at linear colliders within the general 2HDM: Quantum effects and triple Higgs boson self-interactions, Phys. Rev. D 81, 033003 (2010).

[98] T. Plehn, M. Spira, and P. M. Zerwas, Pair production of neutral Higgs particles in gluon-gluon collisions, Nucl. Phys. B479 (1996) 46; Erratum, Nucl. Phys. B531, 655(E) (1998).

[99] R. V. Harlander, S. Liebler, and H. Mantler, SusHi: A program for the calculation of Higgs production in gluon fusion and bottom-quark annihilation in the Standard Model and the MSSM, Comput. Phys. Commun. 184, 1605 (2013).

[100] A. M. Sirunyan et al. (CMS Collaboration), Search for Higgs boson pair production in the $\gamma \gamma \mathrm{b} \bar{b}$ final state in $\mathrm{pp}$ collisions at $\sqrt{s}=13 \mathrm{TeV}$, Phys. Lett. B 788, 7 (2019).

[101] M. Aaboud et al. (ATLAS Collaboration), Search for pair production of Higgs bosons in the $b \bar{b} b \bar{b}$ final state using proton-proton collisions at $\sqrt{s}=13 \mathrm{TeV}$ with the ATLAS detector, J. High Energy Phys. 01 (2019) 030.

[102] M. Aaboud et al. (ATLAS Collaboration), Search for a heavy Higgs boson decaying into a $Z$ boson and another heavy Higgs boson in the $\ell \ell b b$ final state in $p p$ collisions at $\sqrt{s}=13 \mathrm{TeV}$ with the ATLAS detector, Phys. Lett. B 783, 392 (2018). 